\title{
Prevalence of hypomagnesemia in patients undergoing gynecological oncology surgery in tertiary care cancer institute of India
}

\author{
Bikram Bhardwaj*, Ava Dipan Desai, Bijal Manish Patel, \\ Chetna Deepal Parekh, Shilpa Mukesh Patel
}

\begin{abstract}
Department of Gynecologic Oncology, Gujarat Cancer and Research Institute Civil Hospital Campus, Asarva
\end{abstract} Ahmedabad, Gujrat, India

Received: 13 September 2017

Accepted: 16 September 2017

\section{*Correspondence:}

Dr. Bikram Bhardwaj,

E-mail: bikrambhardwaj@gmail.com

Copyright: () the author(s), publisher and licensee Medip Academy. This is an open-access article distributed under the terms of the Creative Commons Attribution Non-Commercial License, which permits unrestricted non-commercial use, distribution, and reproduction in any medium, provided the original work is properly cited.

\begin{abstract}
Background: Hypomagnesemia is an important but unknown risk factor for post-operative complications in patients undergoing surgery for presumed gynecological malignancy. This study aims to evaluate the prevalence of hypomagnesemia in patients undergoing surgery for presumed gynecological cancers referred to our tertiary care Cancer Institute.

Methods: This is a prospective observational study of 100 patients admitted with provisional diagnosis of malignancy. They underwent surgery in one of the Gynecologic Oncology units at The Gujarat Cancer Research Institute, Ahmedabad from October 2016 to April 2017. Hypomagnesemia was defined a serum magnesium levels less than $1.8 \mathrm{mg} / \mathrm{dl}$.

Results: The incidence of pre-operative hypomagnesemia in the entire cohort was $35 \%$. Sixty three percent patients had normal pre-operative magnesium levels and hypermagnesemia was seen in $2 \%$ of study population. Patients with benign disease had $29.6 \%$ pre-operative hypomagnesemia compared with $39.6 \%$ in patients with gynecologic malignancy. Pre-operative hypomagnesemia and even falling levels in post-operative period are an important predictive marker for post-operative complications like increased post-operative pain, post-operative ileus, hypertension and even post-operative hypokalemia. Age, body mass index, hematocrit, surgical indication and length of hospital stay were not associated with hypomagnesemia. Patients undergoing neo-adjuvant chemotherapy before surgery had significant incidence of hypomagnesemia both pre-operatively and post-operatively.

Conclusions: Hypomagnesemia is quite prevalent in patients of gynecologic-oncology undergoing surgery. Preoperative hypomagnesemia and even falling levels in post-operative period have a bearing on the final surgical outcome. Hence pre-operative and post-operative magnesium levels may be included as a valuable marker in all patients undergoing surgery for gynecologic malignancy.
\end{abstract}

Keywords: Gynec-oncology surgery, Hypomagnesemia, Hypokalemia, Post-operative complications

\section{INTRODUCTION}

Magnesium is the second most abundant element inside human cells and the fourth most abundant positively charged ion in the human body. Magnesium is crucial to more than 300 enzyme driven biochemical reactions in the body on a near constant basis. ${ }^{1}$

Magnesium is one of the most common co-factors in the body. Its presence is crucial to 
- Glucose and fat breakdown

- DNA and RNA synthesis

- Regulation of cholesterol synthesis

- Production of proteins and enzymes and

- anti-oxidants like glutathione

Without magnesium we could not produce energy and our muscles would go into a permanent state of contraction. It also plays a pivotal role in the reactions that generate and use ATP, the fundamental unit of energy within the cells of the body. Magnesium is a macro-mineral which unlike trace minerals is required by the body in large amounts. The average human body contains 25 grams of magnesium, one of the six essential elements which must be supplied in diet.

Hypomagnesemia is an electrolyte disturbance in which there is low level of magnesium in the blood. Normal magnesium levels are between 1.8-2.5 mg/dl, with levels less than $1.8 \mathrm{mg} / \mathrm{dl}$ defining hypomagnesemia. Magnesium deficiency is not uncommon in hospitalized patients. Twelve percent of admitted cancer patients and as high as $60-65 \%$ of patients in intensive care units have hypomagnesemia. ${ }^{2-4}$ Thirty percent alcoholics and $25 \%$ diabetic patients have hypomagnesemia. Hypomagnesemia is probably under-diagnosed as testing for serum magnesium levels is not done routinely. Symptomatic hypomagnesemia is commonly associated with hypokalemia, hypocalcemia and metabolic alkalosis. Correction of hypokalemia is challenging without correcting hypomagnesemia. ${ }^{5}$ A number of medical conditions and interventions associated with hypomagnesemia include ${ }^{8,9}$

- Major surgery requiring bowel preparation with sodium phosphate enema.

- Chemotherapy especially platinum compounds

- Diabetes and obesity

- Drugs like diuretics, gentamycin, digitalis, cyclosporine and long term use of proton pump inhibitors

- Trauma, infection, sepsis Transfusion of blood products preserved with citrate

- Alcoholism, starvation and malnutrition.

Malnutrition occurs upto $20 \%$ in gynecologic oncology patients with maximum incidence in ovarian cancers. Patients undergoing surgery for gynecological cancer undergo a number of interventions and have associated medical conditions which cause hypomagnesemia. Hence, the present study aims to evaluate the prevalence and risk factors associated with hypomagnesemia in patients under-going surgery for gynecological malignancy.

\section{METHODS}

This is a prospective study of 100 patients who underwent surgery in one of the Gynaecologic- Oncology unit of The Gujarat Cancer and Research Institute, Ahmedabad for suspicion of malignancy. The present study aims to evaluate the prevalence and risk factors for hypomagnesemia in patients undergoing surgery for gynecological malignancy between October 2016 to April 2017.

\section{Inclusion criteria}

All patients undergoing major surgery for suspicion of gynecological malignancy from October 2016 to April 2017. Major surgery included abdominal hysterectomy, exploratory laparotomy, radical hysterectomy, radical vulvectomy and tumor de-bulking.

\section{Exclusion criteria}

All patients with gynecological malignancy during same time period undergoing other treatment modalities like radiotherapy and chemotherapy. Patients given sodium phosphate enema for bowel preparation were also excluded from the study. Patients undergoing minor surgery during same time period were excluded from the study.

Patients were reviewed for age, BMI, surgical indication, type of surgery, pre-operative and post-operative laboratory values, pre-operative and postoperative pain and medical history of diabetes and hypertension. Patients were included in analysis as having malignancy whether diagnosed pre-operatively or post-operatively. Sodium phosphate enema was not used for bowel preparation in any of the cases.

Pre-operative and post-operative sodium, potassium, calcium and magnesium were assessed. Pre-operative laboratory analysis occurred within 2 weeks of surgery and post operative analysis was done on day1. Sodium (135-145 meq/L), potassium (3.5-5.2meq/L), calcium (9$10.2 \mathrm{mg} / \mathrm{dl})$ and magnesium (1.8-2.5 $\mathrm{mg} / \mathrm{dl})$ were considered normal. The incidence of pre-operative and post-operative hypomagnesemia was assessed. Pain scores were graded from 0 to 10 using Universal Pain Assessment Tool.

Pre-operative pain assessment was done on the day of admission for surgery and post-operative pain scores were obtained during morning rounds on day 1 of surgery. All patients underwent combined epidural and general anaesthesia and received post-operative fluid resuscitation with lactated Ringers solution. Postoperative electrolyte deficiencies were corrected intravenously on day1. Age, BMI, medical history, surgery type, length of stay and post-operative pain scores were assessed for association with hypomagnesemia. Due to very less incidence of hypermagnesemia and aim to assess the impact of hypomagnesemia patients with normal magnesium levels and hypermagnesemia were combined to analyse the risk factors. 


\section{Statistical analysis}

For demographic data comparing 3 groups according to magnesium values, analysis of variance was used for continuous variables and $\mathrm{X}^{2}$ or Fischer exact test was used for categorical variables. For other data to compare hypomagnesemia to normal and hpermagnesemia combined, $\mathrm{t}$ test was used for continuous variables and $\mathrm{X} 2$ or Fischer exact test was used for categorical variables.

\section{RESULTS}

One hundred patients were enrolled for surgery with suspicion of malignancy from October2016 to April 2017. Mean age of the study population under study was 49.5 years (range $15-76$ years).

Ninety patients were vegeterians (90\%). Nine patients $(09 \%)$ had history of infertility and five patients were unmarried. Sixty patients were postmenopausal and 40 patients were premenopausal.

Mean BMI of the study population was 22.2 (Range 1732). Co-morbidities in the form of diabetes, hypertension, hypothyroidism and coronary artery disease were present in 14,26,4 and 3 patients respectively in the study population. Thirty patients were subjected to neoadjuvant chemotherapy before undergoing surgery for gynecological malignancy. Out of 100 patients, final histology was malignant in 69 cases, 25 cases had benign histology and remaining 6 cases had pseudo-tumors.
Table 1: Clinical profile of women undergoing surgery for provisional diagnosis of gynecological malignancy $(\mathrm{n}=\mathbf{1 0 0})$.

\begin{tabular}{|ll|}
\hline Variable & Number (\%) \\
\hline Age-median (range) yrs & $49.5(15-76)$ years \\
\hline Menstrual status & \\
\hline Premenopausal & $40(40 \%)$ \\
\hline Postmenopausal & $60(60 \%)$ \\
\hline Vegeterians & $90(90 \%)$ \\
\hline Mean BMI & $22.2(15-76) \mathrm{Kg} / \mathrm{m}^{2}$ \\
\hline Co-morbidities & \\
\hline Diabetes & $26(26 \%)$ \\
\hline Hypertension & $14(14 \%)$ \\
\hline Hypothyroidism & $4(4 \%)$ \\
\hline Coronary artery disease & $4(4 \%)$ \\
\hline Pre-operative Magnesium & \\
\hline$<1.8 m g / d l$ & $35(35 \%)$ \\
\hline$>1.8 m g / d l$ & $65(65 \%)$ \\
\hline Post-operative Magnesium & \\
\hline$<1.8 m g / d l$ & $64(64 \%)$ \\
\hline$>1.8 m g / d l$ & $36(36 \%)$ \\
\hline Neo-adjuvant chemotherapy & $30(30 \%)$ \\
\hline
\end{tabular}

The prevalence of hypomagnesemia among the study population undergoing gynecologic surgery was $35 \%$ preoperatively $\quad(\mathrm{p}<0.001)$ and $64 \%$ post-operatively ( $\mathrm{p}<0.001$ ). Among patients with benign disease $29.6 \%$ had pre-operative hypomagnesemia compared with $39.6 \%$ of patients with gynecological malignancy.

Table 2: Patient demographics.

\begin{tabular}{|c|c|c|c|c|c|}
\hline Variable & Hypomagnesemia & Normal & Hypermagnesemia & Total & P value \\
\hline All patients (pre-operative) & 35 & 63 & 02 & 100 & $<0.0001$ \\
\hline All patients (post-operative) & 65 & 34 & 01 & 100 & $<0.0001$ \\
\hline Mean Age (years) & 51years & 49years & 53.5years & 52 & 0.98 \\
\hline Average BMI $\left(\mathrm{Kg} / \mathrm{m}^{2}\right)$ & 23.1 & 25.1 & 22.5 & 24.4 & 0.93 \\
\hline \multicolumn{6}{|l|}{ Malignancy } \\
\hline Yes & 51 & 17 & 01 & 69 & \multirow{3}{*}{0.012} \\
\hline No & 13 & 17 & 01 & 31 & \\
\hline Total & 64 & 34 & 02 & 100 & \\
\hline \multicolumn{6}{|l|}{ Cancer type } \\
\hline Uterine & 02 & 02 & 0 & 04 & \multirow{7}{*}{0.85} \\
\hline Cervical & 04 & 03 & 0 & 07 & \\
\hline Ovarian & 38 & 21 & 01 & 60 & \\
\hline Vulvar & 04 & 06 & 0 & 10 & \\
\hline Endometrium & 10 & 08 & 01 & 19 & \\
\hline Uterine & 02 & 02 & 0 & 04 & \\
\hline Total & 58 & 40 & 02 & 100 & \\
\hline
\end{tabular}

The median age was 51 years for patients with preoperative hypomagnesemia and 49 years for patients with normal pre-operative magnesium levels $(\mathrm{P}=0.98)$. The median BMI for patients with hypomagnesemia before surgery was $23.1 \mathrm{Kg} / \mathrm{m}^{2}$ and $25.1 \mathrm{Kg} / \mathrm{m}^{2}$ for patients with normal pre-operative magnesium levels $(\mathrm{P}=0.93)$. 
Table 3: Variables for pre-operative hypomagnesemia.

\begin{tabular}{|c|c|c|c|c|}
\hline & $\mathrm{Mg}<1.8 \mathrm{mg} / \mathrm{dl}$ & $\mathrm{Mg}>1.8 \mathrm{mg} / \mathrm{dl}$ & Total & P value \\
\hline \multicolumn{5}{|l|}{ Cancer Type } \\
\hline Ovarian cancer & $21(35 \%)$ & $39(65 \%)$ & 60 & \multirow{5}{*}{0.776} \\
\hline Cervical cancer & $03(42.8 \%)$ & $04(57.2 \%)$ & 07 & \\
\hline Uterine cancer & $01(25 \%)$ & $03(75 \%)$ & 04 & \\
\hline Endometrial cancer & $08(42.1 \%)$ & $11(57.9 \%)$ & 19 & \\
\hline Vulvar cancer & $02(20 \%)$ & $08(80 \%)$ & 10 & \\
\hline \multicolumn{5}{|l|}{ Co-morbidities } \\
\hline \multicolumn{5}{|l|}{ Hypertension } \\
\hline Yes & $09(25.7 \%)$ & $13(20 \%)$ & 22 & \multirow{3}{*}{0.68} \\
\hline No & $26(74.3 \%)$ & $52(80 \%)$ & 78 & \\
\hline Total & 35 & 65 & 100 & \\
\hline \multicolumn{5}{|l|}{ Diabetes } \\
\hline Yes & $07(20 \%)$ & $08(12.3 \%)$ & 15 & \multirow{2}{*}{0.46} \\
\hline No & $28(80 \%)$ & $57(87.7 \%)$ & 85 & \\
\hline \multicolumn{5}{|l|}{ Both DM, HTN } \\
\hline Yes & $05(14.2 \%)$ & $01(1.5 \%)$ & 06 & \multirow{2}{*}{0.055} \\
\hline No & $30(85.8 \%)$ & $64(98.5 \%)$ & 94 & \\
\hline \multicolumn{5}{|l|}{ Potassium } \\
\hline$<3.6 \mathrm{mg} / \mathrm{dl}$ & $06(17.1 \%)$ & $04(6.1 \%)$ & 10 & \multirow{3}{*}{0.018} \\
\hline $3.6-5.2 \mathrm{mg} / \mathrm{dl}$ & $28(80 \%)$ & $60(92.3 \%)$ & 88 & \\
\hline$>5.2 \mathrm{mg} / \mathrm{dl}$ & $01(2.9 \%)$ & $01(1.6 \%)$ & 02 & \\
\hline Total & 35 & 65 & 100 & \\
\hline
\end{tabular}

Table 4: Variables for post-operative hypomagnesemia.

\begin{tabular}{|c|c|c|c|c|}
\hline Cancer type & $\mathrm{Mg}<1.8 \mathrm{mg} / \mathrm{dl}$ & $\mathrm{Mg}>1.8 \mathrm{mg} / \mathrm{dl}$ & Total & P value \\
\hline Ovarian cancer & 39 & 21 & 60 & \multirow{5}{*}{0.432} \\
\hline Cervical cancer & 05 & 02 & 07 & \\
\hline Uterine cancer & 02 & 02 & 04 & \\
\hline Endometrial cancer & 14 & 05 & 19 & \\
\hline Vulvar cancer & 04 & 06 & 10 & \\
\hline \multicolumn{5}{|l|}{ Co-morbidities } \\
\hline \multicolumn{5}{|l|}{ Hypertension } \\
\hline Yes & $17(26.5 \%)$ & $05(13.8 \%)$ & 22 & \multirow{3}{*}{0.20} \\
\hline No & $47(73.5 \%)$ & $31(86.2 \%)$ & 78 & \\
\hline Total & 64 & 36 & 100 & \\
\hline \multicolumn{5}{|l|}{ Diabetes } \\
\hline Yes & $10(15.6 \%)$ & $04(11.1 \%)$ & 14 & \multirow{3}{*}{0.048} \\
\hline No & $54(84.3 \%)$ & $32(88.9 \%)$ & 86 & \\
\hline Total & 64 & 36 & 100 & \\
\hline \multicolumn{5}{|l|}{ Both DM, HTN } \\
\hline Yes & 05 & 01 & 06 & \multirow{3}{*}{0.414} \\
\hline No & 59 & 35 & 94 & \\
\hline Total & 64 & 36 & 100 & \\
\hline \multicolumn{5}{|c|}{ Lab data Hematocrit } \\
\hline $27-31$ & 54 & 32 & 86 & \multirow[t]{3}{*}{0.76} \\
\hline $31.1-35$ & 10 & 04 & 14 & \\
\hline Total & 64 & 36 & 100 & \\
\hline \multicolumn{5}{|l|}{ Potassium } \\
\hline$<3.6 \mathrm{mg} / \mathrm{dl}$ & 23 & 06 & 29 & \multirow{4}{*}{0.047} \\
\hline $3.6-5.2 \mathrm{mg} / \mathrm{dl}$ & 39 & 30 & 69 & \\
\hline$>5.2 \mathrm{mg} / \mathrm{dl}$ & 02 & 0 & 02 & \\
\hline Total & 64 & 36 & 100 & \\
\hline
\end{tabular}


Malignancy was associated with pre-operative and postoperative hypomagnesemia $(\mathrm{P}=0.012)$. However, cancer type was not indicative of hypomagnesemia among patients with malignancy before surgery $(\mathrm{P}=0.776)$.
Hypertension alone was not associated with pre-operative hypomagnesemia $(\mathrm{P}=0.68)$ whereas it was associated with hypomagnesemia if patients also had diabetes $(\mathrm{P}=$ 019).

Table 5: Effect of neo-adjuvant chemotherapy (NACT) on magnesium levels.

\begin{tabular}{|c|c|c|c|c|}
\hline Pre-operative $\mathrm{Mg}>1.8 \mathrm{mg} / \mathrm{dl}$ & Post-operative $\mathrm{Mg}<1.8 \mathrm{mg} / \mathrm{dl}$ & Post-operative $\mathrm{Mg}>1.8 \mathrm{mg} / \mathrm{dl}$ & Total & P value \\
\hline 15 & 21 & 09 & 100 & \multirow{2}{*}{0.04} \\
\hline 50 & 43 & 27 & 100 & \\
\hline
\end{tabular}

Diabetes alone was not associated with pre-operative hypomagnesemia $(\mathrm{P}=0.46)$. Surgical indication and classification were not associated with pre-operative hypomagnesemia $(\mathrm{P}=0.776)$

Pre-operative hematocrit levels had no association with pre-operative hypomagnesemia $(\mathrm{P}=0.055)$. Thirty patients in our study were exposed to neo-adjuvant chemotherapy before undergoing surgery. $15(50 \%)$ had pre-operative magnesium levels less than $1.8 \mathrm{mg} / \mathrm{dl}$ whereas $21(70 \%)$ had post-operative hypomagnesemia.

Table 6: Peri-operative outcome associated with hypomagnesemia- post-operative.

\begin{tabular}{|lccl|}
\hline Variable & $\mathrm{Mg}<1.8 \mathrm{mg} / \mathrm{dl}$ & $\mathrm{Mg}>1.8 \mathrm{mg} / \mathrm{dll}$ & $\begin{array}{l}\mathrm{P} \\
\text { value }\end{array}$ \\
\hline $\begin{array}{l}\text { Average } \\
\text { length of } \\
\text { stay }\end{array}$ & 7.6 & 6.25 & 0.60 \\
\hline Pain score & 3.4 & 2.4 & 0.049 \\
\hline
\end{tabular}

Post-operative hypomagnesemia had a strong association with major surgery, exposure to chemotherapy $(\mathrm{P}=0.04)$, diabetes $(\mathrm{P}=0.048)$ and hypokalemia $(\mathrm{P}=0.047)$. Surgical indication was not associated with post-operative hypomagnesemia $(\mathrm{P}=0.432)$. There was no direct association of post-operative hypomagnesemia with hypertension $(\mathrm{P}=0.20)$ and post-operative haematocrit $(\mathrm{P}=.41)$. Diabetes had a significant association with postoperative hypomagnesemia $(\mathrm{P}=0.048)$.

Hypomagnesemia had an association with pre-operative and post-operative pain $(\mathrm{P}=0.048)$ but not with average length of stay $(\mathrm{P}=0.60)$. However, very low levels of magnesium less than $1 \mathrm{mg} / \mathrm{dl}$ had a strong association with prolonged intensive care unit stay and even requirement of ventilatory support adding to the length of stay in the hospital. Thirteen patients in our study had post-operative ileus. Of these 11 patients had hypomagnesemia.

Five patients had new onset hypertension with associated hpomagnesemia in 4 cases. Aggravation of pre-existing hypertension is seen in 10 cases with associated hpomagnesemia in 8 of them. Five patients were admitted in intensive care unit requiring ventilator support. Of these 4 patients had critically low magnesium levels.

Table 7: Prevalence of hypomagnesemia according to tumor type.

\begin{tabular}{|lll|}
\hline Tumor type (Total) & $\mathrm{Mg}<1.8 \mathrm{mg} / \mathrm{dl}$ & $\mathrm{Mg}>1.8 \mathrm{mg} / \mathrm{dl}$ \\
\hline Malignant (69) & $51(73.9 \%)$ & $18(26.1 \%)$ \\
\hline Benign (25) & $09(36 \%)$ & $16(64 \%)$ \\
\hline Pseudo-tumor (6) & $04(66.6 \%)$ & $02(33.4 \%)$ \\
\hline
\end{tabular}

\section{Malignant tumors}

Out of total 100 patients, 69 patients had final malignant histology. Fifty one patients with malignant histology had post-operative hypomagnesemia. Major post-operative complications in the form of post-operative ileus occurred in 10 patients.

Ten patients had new onset hypertension detected intraoperatively requiring anti- hypertensive intra-operatively and in the immediate post-operative period and 5 patients had aggravation of pre-existing hypertension.

Three patients with magnesium levels less than $1 \mathrm{mg} / \mathrm{dl}$ had admissions in intensive care unit. Three patients with levels less than $1 \mathrm{mg} / \mathrm{dl}$ had convulsions.

\section{Benign tumors}

Out of total 100 cases, 25 patients had final benign histology. Hypomagnesemia was present in 9 patients. Two patients had post-operative ileus and 1 patient had very low magnesium levels and was admitted in ICU with aggravation of pre-existing coronary artery disease requiring ventilatory support.

\section{Pseudo-tumors}

There were 6 cases of pseudo-tumors in our study. Hypomagnesemia was present in 4 of them. 1 patient had post-operative ileus and 1 patient had aggravation of preexisting hypertension. 
Table 8: Common post-operative complications and their incidence according to tumor type.

\begin{tabular}{|lllll|}
\hline Complications (No.) & Malignant & Benign & Pseudo-tumor & $\mathbf{M g}<\mathbf{1 . 8 m g / d l}$ \\
\hline Ileus (13) & 10 & 02 & 01 & 11 \\
\hline New onset HTN (5) & 03 & 02 & 0 & 04 \\
\hline Aggravation of HTN (10) & 07 & 02 & 01 & 08 \\
\hline Convulsions (3) & 03 & 0 & 0 & 03 \\
\hline ICU admission and ventilatory support (5) & 03 & 01 & 01 & 04 \\
\hline
\end{tabular}

\section{DISCUSSION}

Magnesium is a mineral utilized by each organ as a part of our body, particularly heart muscles and kidneys. The total body stores of magnesium are between 21 and 28 grams in an average $70 \mathrm{~kg}$ man. Normal magnesium levels are 1.8 to $2,5 \mathrm{mg} / \mathrm{dl}$. Most of the magnesium level in the body is in the skeletal bony mass which accounts for more than $50 \%$ of the body stores. The root-cause behind the ill effects of unexplained exhaustion, strange heart rythms or even muscle fits and eye jerks lies in the low magnesium levels. Early signs of magnesium deficiency include loss of apetite, headache, nausea, fatigue and weakness. Serious manifestations of hpomagnesemia include seizures, coronary spasms, abnormal heart rythms and personality changes. A study published in the American Journal of Clinical Nutrition revealed that higher intake of dietary magnesium was connected with lower risk of colorectal tumors. Vegetables like spinach, beans, nuts, seeds, avocados, pumpkin and sunflower are rich sources of magnesium. About $30-40 \%$ of dietary magnesium is absorbed principally in jejunum and ileum and is stimulated by Vitamin D.

Hypomagnesemia has been linked to poor outcome in several different patient populations. In a study of nearly 21000 patients on maintenance dialysis, patients with lowest magnesium levels were at highest risk for death. Hypomagnesemia is a common development in critically ill sepsis patients and indicates poor prognosis. There is a significant association between hypomagnesemia and increased need for mechanical ventilation, prolonged intensive care unit stays and increased mortality in this subset of population based on observational studies. In a Mayo Clinic review of 65,974 hospitalised adult patients hypomagnesemia on admission was associated with increased hospital mortality. Death rates were $2.2 \%$ in patients with magnesium levels of $1.5-1.69 \mathrm{mg} / \mathrm{dl}$ and $2.4 \%$ in those with levels below $1.5 \mathrm{mg} / \mathrm{dl}$ as compared to $1.8 \%$ in patients with levels of $1.7-1.89 \mathrm{mg} / \mathrm{dl}$. The present study reveals hypomagnesemia is prevalent both pre-operatively(35\%) and post-operatively $(64 \%)$ in a significant proportion of patients undergoing surgery for gynecological cancer. ${ }^{4,10,11}$ Even falling levels of serum magnesium in post-operative period has a bearing on the final surgical outcome. Falling serum magnesium levels indicate the magnitude of surgical stress which has a positive impact on post-operative complications.
Prevalence of hypomagnesemia is more after surgery similar to other studies evaluating serum magnesium in post-operative period. ${ }^{2}$ In present study the prevalence of post-operative hypomagnesemia was $64 \%$. Hypomagnesemia was associated with major surgery especially abdominal surgery in patients cared for by surgical oncologists. ${ }^{7,9,12}$ Schwartz et al demonstrated significant decreases in potassium, calcium, and phosphate along with hypomagnesemia in patients after laparotomy. ${ }^{12}$ In present study hypomagnesemia was associated with hypokalemia in $23 \%$ and hypocalcemia in $64 \%$ cases respectively in post-operative period. Schwarz et al also found that hypomagnesemia did not increase length of hospital stay, although other studies concluded a longer length of stay in critically ill patients with hypomagnesemia. ${ }^{13,14}$ In present study the average length of stay in patients with post-operative hypomagnesemia was7.6 days which was in comparison with patients having normal magnesium levels (6.25 days).

The significantly higher preoperative and postoperative pain scores that we report in patients with a low magnesium level correlate to previous research on the potential analgesic benefit of magnesium, although this theory is represented by conflicting results in the literature. Tramer et al found that patients had less postoperative analgesic requirement and experienced a better quality of sleep when given peri-operative magnesium sulfate. This effect has, however, not been universally replicated or recognized. In present study, there was a significant increase of pain score to 3 .4in patients with hypomagnesemia compared to 2.4 in patients with normal magnesium levels. In a systematic review, Lysakowski et al found that 4 studies concluded a significant fall in pain killer requirements and pain intensity with peri-operative magnesium supplementation, whereas 4 other studies showed no such difference in either outcome. ${ }^{15}$ The effect of postoperative magnesium repletion has been evaluated and is, however, likely that serum magnesium is an identifiable marker of a more complex underlying problem resulting in decreased pain tolerance.

Consequences of hypomagnesemia can range from mild to severe. Electrolyte imbalances associated with hypomagnesemia include hypokalemia, hypocalcemia, hypoalbuminemia, and hypophosphatemia., ${ }^{4,5,16}$ In present study there was associated hypokalemia (23\%) and hypocalcemia $(64 \%)$ along with hypomagnesemia $(64 \%)$ in post-operative period. Severe hypomagnesemia 
$(<1.0 \mathrm{mEq} / \mathrm{dL})$ in intensive care patients is associated with a higher mortality rate, need for ventilatory support, and prolonged duration of mechanical ventilation. ${ }^{5,16}$ In current study 3 patients had magnesium levels less than $1 \mathrm{mg} / \mathrm{dl}$. Two of these patients had convulsions and 1 patient had ECG abnormality in the form of atrial fibrillations. All 3 patients were admitted in intensive care unit and required ventilatory support. Severe hypomagnesemia has also been associated with torsades de pointes. ${ }^{18}$ Magnesium deficiency has also been postulated to play a role in the development of myocardial infarction. ${ }^{19}$ One study even suggests that routine serum magnesium levels may identify patients at risk for myocardial infarction. ${ }^{20}$ In present study none of the patients with hypomagnesemia had myocardial infarction. However, 2 patients in present study with severe hypomagnesemia had cardiac decompensation in the form of atrial fibrillations and acute left ventricular failure respectively.

Hypokalemia is a common event in patients with hypomagnesemia, occurring in 40 to $60 \%$ of cases. In present study, the incidence of hypokalemia with associated hypomagnesemia was $23 \%$. This is partly due to underlying disorders that cause magnesium and potassium losses including diuretic therapy and diarrhea. The earliest manifestation of hypomagnesemia include neuromuscular irritability in the form of tetany, Chvosteks and Trousseaus signs and convulsions. The threshold of axon stimulation is decreased and nerve conduction velocity is increased when serum magnesium is reduced, leading to an increase in excitability of muscles and nerves. Two patients with very low magnesium levels in post-operative period had convulsions.

Hypomagnesemia is now a recognized cause of cardiac arrhythmias. ECG changes include prolongation of conduction and non specific ST depression. Patients with hypomagnesemia are particularly susceptible to digoxin related arrhythmias. One patient in our study had atrial fibrillations. The incidence of cardiac arrhythmias also correlate with degree of magnesium deficiency in patients with coronary artery disease. Very low magnesium levels can cause torsades de pointes. ${ }^{18}$ Magnesium supplementation may reduce the frequency of potentially fatal ventricular arrhythmias. It has been suggested that magnesium plays a role in blood pressure regulation. Hypertension appears to be uniformly characterized by a decrease in intracellular free magnesium that, due to increased vascular tone and reactivity causes an increase in total peripheral resistance. Magnesium deficiency is also known to be accompanied by thrombotic tendencies, increased platelet aggregation and increased coronary artery responsiveness to contractile stimuli. These factors are important in initiation of acute myocardial infarction. ${ }^{19}$ Research is conflicting regarding the benefits of intravenous administration of magnesium in the setting of acute myocardial infarction. In present study, we had 10 cases where there was aggravation of pre-existing hypertension associated with hypomagnesemisa in postoperative period requiring further dose adjustments of anti-hypertensives. Five cases had new onset hypertension associated with hypomagnesemia requiring some anti-hypertensive treatment intra-operatively and in the immediate post-operative period. Hypomagnesemia has a lot of bearing on the post-operative outcome. Postoperative ileus is one of the major complications in the immediate post-operative period associated with hypomagnesemia. In our study 13 patients had postoperative ileus. Eleven of these patients had hypomagnesemia and 8 patients had concomitant hypomagnesemia along with hypokalemia.

Main limitation of this study is the small size of study population. Another limiting factor is not stratifying surgical procedures into routine and complex surgeries. More intensive cases such as debulking procedures and bowel anastomosis including confounding factors such as prolonged operative time, peri-operative morbidity and blood loss were not taken into account in this study

\section{CONCLUSION}

Hypomagnesemia is a lesser known risk factor for postoperative complications in patients with gynecological malignancies. The present study reveals hypomagnesemia is prevalent both pre-operatively and post-operatively in a significant proportion of patients undergoing surgery with suspicion of gynecological malignancy. Even falling levels of serum magnesium in post-operative period have a bearing on the fina surgical outcome. Female patients undergoing gynecological surgery are more prone to electrolyte imbalance because of associated co-morbid conditions like diabetes and hypertension and use of certain medications like diuretics and proton pump inhibitors by these patients. Serum magnesium is a relatively low cost test which should be utilized more frequently as a prognostic tool to reduce the risk of adverse surgical outcomes. The impact of pre-operative nutrition in patients suffering hypomagnesemia and its impact on post-operative outcome as well as prognosis in patients undergoing surgery for gynecological cancers needs to be further elucidated.

Funding: No funding sources Conflict of interest: None declared

Ethical approval: The study was approved by the Institutional Ethics Committee

\section{REFERENCES}

1. Jahnen-Dichent W, Ketteler M. Magnesium basics. Clin Kidney J. 2012;5:i3-i14.

2. Wong ET, Rude RK, Singer FR, Shaw Jr ST. A high prevalence of hypomagnesemia and hypermagnesemia in hospitalized patients. Am J Clin Pathol. 1983;79:348-52. 
3. D'Erasmo E. Hypocalcemia and hypomagnesemia in cancer patients. Biomed Pharmacother. 1991;45:3157.

4. Chernow B, Bamberger S, Stoiko M, Vadnais M, Mills S, Hoellerich V et al. Hypomagnesemia in patients in postoperative intensive care. Chest. 1989;95: 391-7.

5. Agus ZS. Hypomagnesemia. J Am Soc Nephrol. 1999;10:1616-22.

6. Konner JA, Grabon DM, Gerst SR, Iasonos A, Thaler H, Pezzulli SD et al. Phase II study of intraperitoneal paclitaxel plus cisplatin and intravenous paclitaxel plus bevacizumab as adjuvant treatment of optimal stage II/III epithelial ovarian cancer. J Clin Oncol. 2011;29: 4662Y4668.

7. Schwarz RE, Zagala-Neverez K. Significant hypomagnesemia after celiotomy: implications of preoperative bowel cleansing with sodium phosphate purgative. Surgery. 2002;131:236.

8. Gau JT, Yang YX, Chen R, Kao TC. Uses of proton pump inhibitors and hypomagnesemia Pharmacoepidemiol. Drug Saf. 2012; 21:553Y559

9. Lecube A, Baena-Fustegueras JA, Fort JM, Pelegrí D, Hernández C, Simó R. Diabetes is the main factor accounting for hypomagnesemia in obese subjects. PLoS One. 2012 Jan 24;7(1):e30599.

10. Place HM, Enzenauer RJ, Muff BJ, Ziporin PJ, Brown CW. Hypomagnesemia in postoperative spine fusion patients. Spine. 1996 Oct 1;21(19):2268-72.

11. Aglio LS, Stanford GG, Maddi R, Boyd JL, Nussbaum S, Chernow B. Hypomagnesemia is common following cardiac surgery. J Cardiothoracic Vasc Anesth. 1991;5:201Y208.

12. Schwarz RE, Nevarez KZ. Hypomagnesemia after major abdominal operations in cancer patients: clinical implications. Arch Med Res. 2005;36:36-41.
13. Safavi M, Honarmand A. Admission hypomagnesemiaV impact on mortality or morbidity in critically ill patients. Middle East J Anesthesiol. 2007; 19:645-660.

14. Kumar S, Honmode A, Jain S, Bhagat V. Does magnesium matter in patients of Medical Intensive Care Unit: a study in rural central India. Indian S Crit Care Med. 2015;19:379-383.

15. Lysakowski C, Dumont L, Czarnetzki C, Tramèr MR. Magnesium as an adjuvant to postoperative analgesia: a systematic review of randomized trials. Anesth Anal. 2007 Jun 1;104(6):1532-9.

16. Limaye CS, Londhey VA, Nadkart MY, Borges NE. Hypomagnesemia in critically ill medical patients. J Assoc Physicians India. 2011 Jan;59(1):19-22.

17. Vallee BL, Wacker WE, Ulmer DD. The magnesium-deficiency tetany syndrome in man. $\mathrm{N}$ Engl J Med. 1960;262:155.

18. Sherwood RA, Aryanayagam P, Rocks BF. Hypomagnesemia in the elderly. Gerontology. 1986;32:105-9.

19. Bloom S. Effects of magnesium deficiency on the pathogenesis of myocardial infarction. Magnesium. 1986;5:154-164.

20. Whang R. Magnesium and potassium interrelationships in cardiac arrythmias. Magnesium. 1986;5:127-133.

Cite this article as: Bhardwaj $\mathrm{B}$, Desai $\mathrm{AD}$, Patel BM, Parekh CD, Patel SM. Prevalence of hypomagnesemia in patients undergoing gynecological oncology surgery in tertiary care cancer institute of India. Int J Reprod Contracept Obstet Gynecol 2017;6:4355-62. 